ditism could be related to variations in testis size.

Several lines of evidence suggest that during embryogenesis, the mammalian gonad may need to grow to a certain size by a given stage of development in order to differentiate into a testis, failing which the gonad becomes an ovary ${ }^{3}$. In populations with small testes, a few $\mathrm{XY}$ individuals may fail to reach the threshold, leading to some ovarian development. On the other hand in populations with large gonads some testicular development may occur in a few $\mathrm{XX}$ individuals.

In both $\mathrm{XX}$ and $\mathrm{XY}$ hermaphrodites, an ovary tends to be situated on the left side and a testis or ovotestis on the right. The bilateral asymmetry of the gonads in true hermaphroditism can be related to an asymmetry of growth in human fetal gonads ${ }^{3}$. It is noteworthy therefore that the same asymmetry is seen in the testes of adults in three population samples ${ }^{1}$, thus providing additional evidence of a relationship between testis size and human hermaphroditism.

Ursula MitTwoch Department of Genetics and Biometry, Wolfson House, 4 Stephenson Way, University College London,

London NW1 $2 H E$, Uk

1. Diamond, J.M. Nature 320, 488-489 (1986)

2. Simpson, J.L. in Sex Differentiation and Chromosomal Abnormalities (eds Summitt, R.L. \& Bergsma, D.) 9-35 (Liss, New York, 1978).

3. Mittwoch, U. Ann. hum. Genet. 50, 103-121 (1986).

\section{Accuracy of testing for Huntington's disease}

SIR-Earlier this year, Nature published two letters ${ }^{1.2}$ giving opposing views on the use of the recently discovered G8 marker for presymptomatic diagnosis of Huntington's disease (HD). We indicated that the "at risk" individual should be provided the opportunity to make an informed choice as to whether he or she wished to be tested but that it was not appropriate to proceed with presymptomatic testing until the question of non-allelic heterogeneity had been adequately addressed. Until further studies being carried out in several centres had been completed, we could not adequately define the accuracy of the presymptomatic test, so that it was impossible to provide the data necessary for an informed choice. Any results would carry a degree of uncertainty that could not at that time be estimated by the genetic counsellor on the basis of existing data. (We were not arguing for a greater level of accuracy in testing, as was suggested ${ }^{3}$, but simply that the level of accuracy being quoted should have a firm basis.)

Linkage studies of large HD pedigrees with the G8 probe have since produced enough data to estimate accurately the possibility of non-allelic heterogeneity. The data, which will be presented at the

\section{Scientific Correspondence}

Scientific Correspondence is intended to provide a forum in which readers may raise points of a rather scientific character. They need not arise out of anything published in Nature, but those that do should not be highly technical comments on Articles or Letters (where the Matters Arising section remains appropriate).

International congress of Human Genetics in Berlin next month, indicate that if a second locus does cause the disease in some families, it is present in at most 10 per cent of HD pedigrees. Using this figure in assessing genetic risk, it is now scientifically justifiable to proceed with presymptomatic diagnosis.

In our previous letter we also suggested that presymptomatic diagnosis in $\mathrm{HD}$ is not comparable to most other medical tests. Healthy "at risk" individuals will be taking a test that will predict their own future, one perhaps dominated by a devastating untreatable disease with both physical and mental effects. The HD gene is known to be associated with psychiatric implications and the risk of suicide is significantly increased. Presymptomatic testing has consequences which may prove damaging both to the individual being tested and to other family members. Clearly the testing should only be performed with considerable counselling and support, both before and after the delivery of the results.

In view of these problems, the Massachusetts General Hospital established the Committee for Presymptomatic Huntington's Disease Testing to discuss the most appropriate method of implementing a testing programme. The committee includes representatives from HD lay organizations, from other institutions and from the New England HD Center, as well as experts in medicolegal issues. The committee members indicated that, as with other new medical procedures, the most responsible course of action would be to begin presymptomatic HD testing as a controlled clinical trial. This would permit investigators to assess the most effective means of delivering the information while closely monitoring and exploring methods to deal with, and possibly prevent, negative consequences. The results obtained would also have implications in many other late-onset neurological and behavioural disorders for which similar linkage tests may soon be developed.

Experimental testing programmes will begin in the autumn of 1986 at the Massachusetts General Hospital, Johns Hopkins University and Columbia University and are also planned at several other centers. We are making the $\mathrm{G} 8$ probe available through the Committee for Presymptomatic Huntington's Disease Testing to any other institutions interested in initiating clinical testing programmes approved by their local institutional review board, or equivalent ethics committee. We believe that this approach will result in a clearer understanding of both the potential positive and negative consequences of presymptomatic HD diagnosis by linkage analysis and will also be in the best interest of "at risk" individuals and their families.

JAmes F. Gusella

Neurogenetics Laboratory,

Massachusetts General Hospital,

Boston, Massachusetts 02114, USA

1. Watt, D.C., Lindenbaum, R.H., Jonasson, J.A. \& Edwards, J.H. Nature 320, 21 (1986)

2. Gusella, J.F. Nature 320, 21-22 (1986)

3. Maddox, J. Nature 320, 11 (1986).

\section{Faster than the eye can see}

SIR-In their otherwise lucid observations of a long-duration lightning flash Baker and Baker-Blocker ${ }^{1}$ say that a 400 ms flash "is too brief to allow interpretation, since the duration of a perceptual experience is around $0.4 \mathrm{~s}$ for the dark-adapted visual system. . ."But this evades the question of what they mean by "interpretation" and "perceptual experience" because two or more transient flashes, clicks, or taps separated by $\geqslant 30 \mathrm{~ms}$ (that is at $>30 \mathrm{~Hz}$ ) are easily detected as an ordered temporal sequence. For mixed sensory modalities, for example click and tap, this interval may be $\geqslant 50 \mathrm{~ms}$, especially when the somatosesnory stimulus precedes the auditory one ${ }^{2}$. Surely such psychophysical judgements, by whatever method obtained, involve "interpretation" (discrimination of the physical parameters of each transient) over intervals briefer than $400 \mathrm{~ms}$.

But I agree that transients repeating faster than about $5-7 \mathrm{~Hz}$ are hard to count verbally and harder still to replicate by motor output, and I take it that this fact somehow lies behind your correspondents' statement. Also, the long duration $(\geqslant 500 \mathrm{~ms})$ of electrocortical field potential resonances and of relevant multiple neural firings following any suprathreshold, impulse-like sensory stimulus is well known from studies on both humans and nonhumans; less well known and accepted is the evidence for the absence of a human report of tactile sensation if the late resonances of a somatosensory evoked potential are experimentally aborted by neurosurgical manoeuvres ${ }^{3}$.

ERBP Laboratory,

120 Nature Creek,

Milledgeville,

Georgia 31061, USA

1. Baker, D.G. \& Baker-Blocker, A. Nature 322, 215 (1986)

2. Stowell, H. in Neuropsychol \& Cognit Vol 2. NATO ASI, MCG, Augusta GA, USA 1980 (eds. Maltaesha, R.N. \& Hartlage, L.C.) 565-589 (Nijhoff, The Hague, 1982).

3. Libet, B., Wright, E.W., Feinstein, B. \& Pearl, D.K. Brain 102, 193-224 (1979) 